\title{
LA COMPILACIÓN DE HUESCA. APUNTES SOBRE EL PROCESO DE REDACCIÓN
}

\author{
THE HUESCA COMPILATION. DRAFTING PROCESS NOTES
}

\section{Miguel Carabias Orgaz IES El Greco. Toledo}

Resumen: En este trabajo se analiza el proceso de elaboración de la Compilatio Minor o Compilación de Huesca a partir de su primitiva redacción en lengua romance, la cual sería posteriormente traducida para conformar la versión latina oficial. Para ello, cotejamos el texto con diversas fuentes de la legislación altoaragonesa inmediatamente anterior, de la que procede en gran medida el texto de los Fueros de Aragón.

Palabras clave: Derecho medieval, Fueros de Aragón, Compilación de Huesca, Fueros de Jaca, legislación altoaragonesa.

\begin{abstract}
In this work we analyze the Compilatio Minor or Compilación de Huesca writing process, from its early composition in the Romance language, and translated afterwards to become the official Latin version. To do that, we compare the text with several sources of the immediately preceding Higth Aragon legislation, from which most of the text of the Fueros of Aragon is derived.
\end{abstract}

Keywords: Medieval law, Fueros of Aragon, Compilación de Huesca, Fueros of Jaca, Higth Aragon legislation.

\section{Los Fueros de Aragón. Primera redacción en lengua romance}

Recientemente daba a conocer la existencia de un documento de mediados del siglo XIII ${ }^{1}$ que contiene parcialmente el texto romance aprobado en las Cortes de Huesca de 1247. Se trata de un folio manuscrito sobre vitela que

1.- Miguel Carabias Orgaz, "Los Fueros de Aragón. Una versión romance de mediados del siglo XIII", Revista de Filología Española, XCIII, 2º julio-diciembre, 2013, pp. 313-326. 
hallé formando parte de la encuadernación de un legajo de mediados del siglo XVI y que doné a la Biblioteca Nacional de España (RES/286/2). El manuscrito presenta unas dimensiones de 150 x $210 \mathrm{~mm}$ y su caja de escritura es de 115 x $176 \mathrm{~mm}$. El texto está copiado a línea tirada, con veintiséis renglones tanto en el recto como en el vuelto, y su contenido se distribuye en diez párrafos, sin rúbricas ni títulos. La letra del documento es gótica textual, con rasgos de la primera época, y el análisis paleográfico ha permitido situarlo cronológicamente en torno al año 1250, es decir, en fecha muy cercana a la redacción de los Fueros de Aragón. Con respecto a la lengua empleada, se pueden identificar algunos rasgos característicos del romance aragonés (la conservación del grupo inicial latino KL-, el posesivo de tercera persona lures o la conservación de -D- intervocálica en determinados contextos).

Aunque recoge un breve pasaje del libro segundo, este documento tiene un extraordinario interés. En primer lugar, porque es el testimonio documental más antiguo de los Fueros de Aragón; en segundo lugar, porque coincide casi puntualmente con la versión latina oficial, tanto en su contenido como en la ordenación de sus capítulos. Es, por tanto, más que probable que se trate del único testimonio hasta ahora conocido de la primera redacción en lengua romance de los Fueros, sobre la cual se llevó a cabo la traducción latina que estuvo vigente hasta el siglo XVIII.

La redacción que se recoge en este manuscrito, a la cual he llamado $C A$, se hizo en lengua vulgar para que pudiera ser comprendida y discutida por todos los asistentes a las cortes. Inmediatamente después fue traducida al latín, que era la lengua de la cancillería, y se le añadieron los títulos o rúbricas correspondientes — de romancio in latinum translatis, et sub debitis titulis collocatis-. Dicha traducción se hizo, además, de forma literal, palabra por palabra, de manera que hoy puede reconocerse con facilidad.

\section{La Compilación de Huesca. Precedentes en la legislación ALTOARAGONESA}

Los Fueros de Aragón de 1247 eran fruto del empeño legislador de Jaime I, quien trataba de fijar un texto que remediara la falta de documentos forales fiables en su reino, dotando además a Aragón de un único código legal que pudieran utilizar los jueces en la resolución de sus pleitos. Para ello, había ordenado reunir cuantos textos recogían la tradicional legislación aragonesa, procediendo a su lectura, selección y corrección, añadiendo luego algunas disposiciones nuevas y, por último, dotando al conjunto de un ordenamiento sistemático: 


\begin{abstract}
...Foros Aragonum, prout ex variis praedecessorum nostrum scriptis collegimus, in nostro fecimus auditorio recitari, quorum singulis collationibus discussa omnia subtilius, et detractis supervecuis et inutilibus, completis minus bene loquentibus, et obscuris competentibus interpretationibus expositis, sub volumine et certis titulis antiquorum Fororum, quosdam ammovimus, correximus, supplevimus, ac eorum obscuritatem elucidavimus, omnium dictarum personarum consilio et convenientia penitus annuente.
\end{abstract}

Ahora bien, ¿cuáles fueron esas fuentes utilizadas y en qué medida se valieron de ellas los juristas de 1247 para confeccionar el nuevo código que conocemos como Fueros de Aragón? ¿De qué manera se adaptaron esos materiales al nuevo código? En resumidas cuentas, ¿qué relación existe entre la Compilación de Huesca y el derecho aragonés anterior?

Es sabido que la Compilación de Huesca se fundamenta en gran medida en la legislación de origen jaqués o altoaragonés precedente, sin embargo ésta ha llegado a nosotros a través de escasos testimonios, en ocasiones fragmentarios y contradictorios entre sí y, en cualquier caso, recogidos en códices materialmente elaborados con posterioridad a 1247.

Sabemos también que Sancho Ramírez concedió un fuero a Jaca, cuya redacción seguramente se remonta al último tercio del $\mathrm{XI}^{2}$, que poco después fue concedido dicho fuero a Sangüesa y Estella y, a lo largo del siglo XII, se dio a diversas poblaciones de Aragón y Navarra: Aínsa, Alquézar, San Cernín de Pamplona, Puente la Reina, etc. Incluso llegó a algunos puntos de la corona de Castilla, como San Sebastián ${ }^{3}$. Pero aquel primer texto foral, muy breve y redactado en latín, había llegado a ampliarse de forma considerable a finales del siglo XII o comienzos del XIII. En primer lugar, en cuanto al espacio geográfico de aplicación, pues ya en 1187, cuando Alfonso II confirmaba los fueros, éstos se extendían mucho más allá de las montañas jacetanas —el antiguo fuero local se había "territorializado"4_. Por otro lado, había llegado a ser un corpus normativo que no se reducía ya a los veinticuatro capítulos de la concesión de Sancho Ramírez, sino que incluía un conjunto de normas procedentes de la tradición, las cuales ya empezaban a ponerse por escrito. Los "foristas" incorporaban las "antiguas costumbres" y utilizaban materiales diversos para elaborar sus propias recopilaciones. Tal como señala Delgado

2.- Antonio Ubieto Arteta, Jaca: documentos municipales, Valencia, Textos Medievales, 1975, pp. 20-21.

3.- Acerca de las relaciones existentes entre estos diversos fueros, puede consultarse Ana Ma ${ }^{\text {a BARRERO }}$ García, "La difusión del Fuero de Jaca en el Camino de Santiago", en El Fuero de Jaca, vol. II, Zaragoza, El Justicia de Aragón, 2003, pp. 113-160.

4.- Jesús Delgado Echeverría, Los Fueros de Aragón, Colección "Mariano de Pano y Ruata", Zaragoza, Caja de ahorros de la Inmaculada de Aragón, 1997, p. 19. 
Echeverría ${ }^{5}$, se había producido una ampliación "por vía consuetudinaria y judicial al amparo de la libertad".

Desde comienzos del siglo XIII, podemos suponer que existía, en palabras de Morales Arrizabalaga ${ }^{6}$, "una tradición jurisprudencial común en el espacio que tiene como capitales a Jaca, Huesca, Zaragoza, Tudela, Pamplona, Estella...". Y, aunque nos encontramos con testimonios escritos en que el fuero recibe diversos nombres, dependiendo del lugar para el que se redactaron, lo cierto es que todos esos testimonios responden a una misma tradición jurídica. Tal como apuntó Delgado Echeverría ${ }^{7}$, "todos estos textos están relacionados entre sî" y —lo que para nosotros es más importante- "resultan imprescindibles para estudiar la Compilación de Huesca".

Sin embargo, como ya he dicho, nos encontramos con recopilaciones elaboradas en el ámbito privado, por parte de "conocedores del fuero" o "foristas", que las utilizaban interesadamente y las mostraban tan sólo cuando podían obtener algún beneficio de ello. No existía aún en Aragón un código con carácter oficial que pudieran utilizar los jueces durante sus pleitos, sino muchas y diversas recopilaciones que no siempre coincidían o que incluso a veces se mostraban contradictorias.

En buena medida, esta falta de documentos fiables — nulla scriptura certa vel autentica - y las malas prácticas de algunos "foristas" motivarán el proyecto legislador de Jaime I y la aprobación, durante las Cortes de Huesca de 1247, de la llamada Compilatio Minor.

A continuación trataremos de identificar algunas conexiones entre aquel derecho tradicional altoaragonés y la Compilación de Huesca, señalando algunas fuentes que pudieron utilizarse en el proceso de elaboración del nuevo código. En este análisis, resulta sumamente revelador el texto conservado de la primera redacción romance $(C A)$, pues aunque se trata de un breve pasaje que recoge diez capítulos o fueros, representa el nexo de unión entre ese derecho tradicional y el texto latino oficial.

5.- Ibídem, p. 20.

6.- Jesús Morales Arrizabalaga, "Los fueros de Sobrarbe como discurso político. Consideraciones de método y documentos para su interpretación", Huarte de San Juan. Revista de Ciencias Humanas y Sociales, Universidad de Navarra, 1 (1994), p. 174.

7.- Jesús Delgado Echeverría, "Fueros de Jaca y Fueros de Aragón. Tablas de concordancias", en El Fuero de Jaca, vol. II, Zaragoza, El Justicia de Aragón, 2003, pp. 163-194. 


\section{Fuentes documentales}

Es posible suponer cierta uniformidad en torno a un cuerpo legal de origen jaqués hacia la primera mitad del siglo XIII, pero lo cierto es que no se han conservado testimonios documentales anteriores a 1247. Además, aunque los manuscritos que conservamos parecen reflejar en muchos aspectos el estado del derecho anterior a las Cortes de Huesca, algunos de ellos pueden haber sido manipulados con posterioridad para acomodarlos a la nueva legislación. Cuando, desde 1247, el derecho aragonés quedaba unificado y todos los fueros anteriores eran derogados, los "foristas" trataron de resistir y mantener sus viejas recopilaciones, pues deseaban ante todo conservar su independencia. Así pues, algunos testimonios manuscritos conservados pueden ser adaptaciones del antiguo derecho altoaragonés en las que fueron eliminados los preceptos no admitidos en 1247, encontrándonos con redacciones enmendadas e interpoladas. En palabras de Canellas, nos hallamos ante un fuero "rejuvenecido".

Un caso paradigmático lo hallamos en el manuscrito que Molho llamó $A^{2}$, donde se inserta, antes del Fuero de Jaca, una serie de 55 capítulos destinados a completar el texto con disposiciones de la Compilación de Huesca. En efecto, todas estas adiciones remiten a pasajes de 1247 que no figuran en la redacción $A$.

Nos encontramos, pues, con una dificultad añadida a la hora de establecer la antigüedad de los testimonios conservados. Podemos datar aproximadamente el manuscrito o el códice que los contiene, sin embargo, como señaló Morales Arrizabalaga, "es arriesgado extrapolar conclusiones desde el continente al contenido". Pese a todo, este mismo autor proponía como testimonios más antiguos los que tienen "aspecto judicial", es decir, los que presentan casos individuales en que los "foristas" podrían haber actuado como árbitros recurriendo a la tradición. Después, con los años, se iría produciendo un proceso de abstracción y generalización. Morales consideró que aquella transformación podía verse especialmente en alguno de los códices editados por Ramos Loscertales 9 .

Por su parte, Molho señaló algunos de los indicios que podrían delatar una mayor antigüedad en las colecciones legislativas aragonesas: un texto menos

8.- Ángel Canellas López, "El cuadernillo foral del Pilar", en Miscelánea ofrecida al Ilmo. Sr. Dr. José María Lacarra y de Miguel, Zaragoza, Facultad de Filosofía y Letras de la Universidad de Zaragoza 1968, p. 128.

9.- Jesús Morales Arrizabalaga, Fueros y Libertades del Reino de Aragón, Rolde de Estudios Aragoneses, Zaragoza, 2007, pp. 22-25. 
desarrollado, la mezcla de redacciones latinas con expresiones romances o el hecho de que no encontremos referencias a un usus terrae.

Teniendo, pues, estas consideraciones a la vista, resumiremos brevemente cuáles son los testimonios documentales con los que contamos. Dejando a un lado el fuero breve otorgado por Sancho Ramírez, que ya dijimos fue redactado seguramente en el último tercio del siglo XI, podemos señalar los siguientes:

- La denominada por Molho redacción $A^{p}$, que recoge tan sólo cuatro capítulos contenidos en un documento del Archivo Municipal de Pamplona. Se trata del traslado de una carta escrita en 1342 por los jurados de Jaca, dirigida a los Veinte de Pamplona para informarles acerca de algunos capítulos del Fuero sobre los que había ciertas dudas. Dichos jurados envían la redacción correcta de los cuatro capítulos, en un latín muy contaminado, tras haber cotejado los ejemplares del Fuero que tenían los navarros con los nuestros antigos et con el padron d'aqueillos. Esto llevó a Molho a suponer que existió un códice original del Fuero y a considerar $A^{p}$ un "vestigio de una redacción arcaica" 10 , poniéndolo en relación con $C P$, a la que también atribuye gran antigüedad.

- La Compilación privada de derecho aragonés $(C P)$, que fue publicada bajo este nombre por Ramos Loscertales ${ }^{11}$. Se recoge en un cuadernillo intercalado en el manuscrito 225 de la Biblioteca de la Universidad de Zaragoza, conocido también como Códice Villarense. Molho destaca su carácter arcaico, que le permite fecharla hacia el segundo tercio del siglo XII, y considera relevante que no haga alusión a un usus terrae concreto y localizable, así como el hecho de que esté redactada en un latín mezclado con formas romances. La pone en relación con $A^{p}$ y se pregunta si podría reflejar una redacción perdida del Fuero de Jaca ${ }^{12}$. También sugiere que tanto $C P$ como $A^{p}$ podrían estar relacionadas con el Fuero de Estella y con la que denomina redacción $Z^{13}$.

10.- Mauricio Molho, El fuero de Jaca, Zaragoza, Consejo Superior de Investigaciones Científicas, Escuela de Estudios Medievales, Instituto de Estudios Pirenaicos, 1964, pp. XXII y XXXI.

11.- José $M^{\mathrm{a}}$ Ramos Loscertales, "Textos para el estudio del derecho aragonés en la Edad Media", Anuario de Historia del Derecho Español, I (1924), pp. 400-408.

12.- Mauricio Molho, "Difusión del derecho pirenaico (Fuero de Jaca) en el reino de Aragón", Boletín de la Real Academia de Buenas letras de Barcelona, XXVIII (1960), pp. 340-343.

13.- M. Molho, "Difusión", p. 307. La redacción $Z$ es la publicada en José Ma Ramos Loscertales, "La observancia 31 «De generalibus privilegiis» del libro VI. Notas para el estudio de su formación", en Homenaje ofrecido a Menéndez Pidal: Miscelánea de estudios lingüísticos, literarios e históricos, vol. III, Madrid, Librería y casa editorial Hernando, 1925, pp. 227-239. 
- La llamada por Molho redacción $A$, que aparece recogida en las versiones $A^{1}, A^{2}$ y $A^{z}$, y es considerada por este autor como "la primera recopilación romanceada extensa del Fuero" 14 , pues aunque los tres manuscritos que la contienen son posteriores a 1247 , recoge el estado del derecho aragonés a comienzos del siglo XIII. La versión $A^{1}$ se halla en un códice de la Biblioteca Nacional de España copiado en el siglo XIV. La versión $A^{2}$ se encuentra en un códice de los Archivos Nacionales de París bajo las siglas J.J.O.O; también fue copiado en el siglo XIV, presenta un texto idéntico al de $A^{1}$, solo que más completo, y viene precedido de una serie de adiciones $(A D)$ que remiten a la Compilación de Huesca. La versión $A^{z}$ fue publicada parcialmente por Lasala ${ }^{15}$ y un siglo más tarde en su totalidad por Ángel Canellas ${ }^{16}$, contiene veintiún capítulos en que se mezcla la lengua romance con expresiones latinas, y Molho ${ }^{17}$ la puso en relación con la redacción $A$, pues coincide con los capítulos 151-171 de $A^{2}$, aunque reconocía variantes que le llevaron a pensar que procedía de un arquetipo diferente al de $A^{1}$ y $A^{2}$. Canellas databa el documento entre los años 1240 y $1270^{18}$.

- La recopilación $U$ forma parte del Códice Villarense de la Universidad de Zaragoza y fue publicada por Ramos Loscertales ${ }^{19}$, quien la consideró "la más antigua forma de redacción del derecho aragonés", adaptada por un jurisconsulto de Zaragoza en el primer tercio del siglo XIII. Reconoció en ella un núcleo principal que relaciona con los Fueros de Jaca, al cual se añaden algunas disposiciones de Borja. Molho, por su parte, la consideraba obra de un "mediocre latinista de Borja", el cual habría traducido un original romance o intensamente romanceado ${ }^{20}$. Más modernamente, el texto ha sido editado de nuevo como "Fueros de Borja y Zaragoza"21, recogiendo algunas adiciones no incluidas por Ramos Loscertales, y se ha propuesto como fecha probable de su redacción el periodo 1144-1155.

- La recopilación $R L$ fue también publicada por Ramos Loscertales ${ }^{22}$. Molho atribuyó su redacción a un clérigo letrado de Huesca, que da

14.- M. MolHo, El fuero de Jaca, p. XXXI.

15.- Manuel Lasala, Examen histórico-foral de la constitución aragonesa, II, Madrid, Impr. Rojas, 1868, pp. 481-495.

16.- A. Canellas, "El cuadernillo", pp. 127-142.

17.- M. MolHo, El fuero de Jaca, pp. XIX-XXI y XXXVII-XL.

18.- A. CANELlas, "El cuadernillo", p. 135.

19.- José Ma Ramos Loscertales, "Textos para el estudio del derecho aragonés en la Edad Media", Anuario de Historia del Derecho Español, II (1925), pp. 491-523.

20.- M. Molho, "Difusión", p. 346

21.- Juan José Morales Gómez y Manuel José Pedraza García, Fueros de Borja y Zaragoza, Zaragoza, Textos Medievales, 1986.

22.- José M M $^{\mathrm{a}}$ Ramos Loscertales, "Textos para el estudio del derecho aragonés en la Edad Media", Anuario de Historia del Derecho Español, V (1928), pp. 390-407. 
un latín pulimentado y más correcto que el de $U$. Se halla en el mismo códice, a continuación de la anterior. Contiene, intercalados, diversos capítulos pertenecientes a la Lex Visigothorum, colecciones canónicas, sentencias de San Isidoro y otros textos. Al igual que $U$, es una copia fragmentaria, materialmente elaborada quizá poco después de 1247, aunque redactada hacia comienzos del siglo XIII.

- La que Molho llamó redacción $B$ se halla recogida en un solo códice del siglo XIV, que se conserva en los Archivos Nacionales de París bajo las siglas J.J.C.C. Molho la consideró una "recopilación iruñense"23, estimando que fue redactada con posterioridad a 1247 , pese a que es la redacción pamplonesa más antigua. A diferencia de la redacción $A$, que es asistemática, Molho observa en $B$ un "esbozo de sistema, rudimentario e incompleto".

- La redacción $S$ fue publicada por Lacarra y Martín Duque ${ }^{24}$ a partir, sobre todo, de un manuscrito de la Biblioteca de la Universidad de Salamanca, que es del siglo XIV. Se trata de otra recopilación pamplonesa derivada del fuero de Jaca, más desarrollada y posterior a $B$.

- Las redacciones $C, D$ y $E$ fueron consideradas por Molho como "redacciones iruñesas sistemáticas" 25 . Intuye este autor un arquetipo único del que derivan todas ellas. $C$ y $E$ presentan algunas diferencias, mientras que $D$ es considerada por Molho una traducción navarro-aragonesa de $C$. La redacción $C$ nos ha llegado en un códice de finales del siglo XV o comienzos del XVI. En cuanto a $D$, se halla en un manuscrito del XIV, al igual que los dos códices que recogen la redacción $E^{26}$. Todas ellas reflejan redacciones posteriores a 1247.

\section{Cotejo de las fuentes. El proceso de Redacción}

Seguidamente, trataremos de poner en relación algunos pasajes significativos de la primitiva redacción romance de la Compilación de Huesca $(C A)$ con diversas fuentes del derecho altoaragonés anterior a 1247. Particularmente relevantes serán, como veremos, las redacciones más próximas en el tiempo a

23.- M. Molho, El fuero de Jaca, p. XL.

24.- José Ma Lacarra y Ángel J. Martín Duque, Fueros de Navarra I. Fueros derivados de Jaca 2. Pamplona, Pamplona, Diputación foral de Navarra e Institución Príncipe de Viana, 1975.

25.- M. MolHo, El fuero de Jaca, p. XLII.

26.- Uno de estos códices, llamado por Molho $E^{2}$, fue publicado en José $\mathrm{M}^{\mathrm{a}}$ Ramos Loscertales, Fuero de Jaca (última redacción), Barcelona, Universidad de Barcelona-Facultad de Derecho, 1927. La edición crítica de las redacciones $C, D$ y $E$ fue publicada, junto con $A$, en M. MolHo, El fuero de Jaca. 
los Fueros de Aragón: la llamada redacción $A$ y las recopilaciones $U$ y $R L$. No nos detendremos a considerar, sin embargo, las variantes que se refieren a la lengua en que están escritos los diversos textos, pues no siempre son claras las fronteras entre los distintos romances, e incluso entre éstos y el latín, que en ocasiones aparecen mezclados. Da la sensación de que nos encontramos con formas híbridas, tal vez con cierto tipo de koiné. Además, la lengua empleada en las obras jurídicas puede reflejar con frecuencia un uso profesional que se aparta del verdadero estado lingüístico de aquella época ${ }^{27}$. Como mucho, podemos suponer un carácter más "oficial" a los textos redactados parcial o totalmente en latín, frente a los textos más claramente romances.

\section{a) Redacciones $A$ y $U$}

En primer lugar, observamos que la redacción de $U$ suele mostrarse menos desarrollada que la de $A$, pues ésta amplía el texto, situándose habitualmente a medio camino entre la redacción latina de $U$ y el texto romance de la Compilación de Huesca $(C A)$ :

(U 32) De homine qui comparat hereditatem uel accipit illam in pignus, fidantie et testes habent esse de illa uilla in qua est hereditas; et quod fidantie uel testes uideant hereditatem. Et si hoc non faciunt, possunt hereditatem perdere qui comparant uel accipiunt in pignus, propter hoc quod nesciunt unde intrant fidantias uel testes, et ille similiter non scit de quo accipit fidantias aut facit testes, et possunt inde exire bono iure.

(A 193) D'om qui compra o pren en peynnora alguna heredat, las fianças e'ls testimonis, segont fuero, deuen esser d'aquela uila on es la heredat, et encara que las fianças ueyan aquela heredat e'ls testimonis atresi que per cert aquelas fianças sapian de qual heredat son fianças e'ls testimonis atresi. E si en tal manera no es feyt, aquel hom qui compra la heredat la pot perdre, o qui la pren pynnora, car no saben dod son las fianças ni los testimonis; et altresi aquel qui compra la heredat o la pren en pynnora no sap de la heredat qui's n'a pres fianças o qui n'a feyt testimonis e asi la pot perdre.

Por su parte, $C A$ presenta una redacción que, estando más cerca de $A$, supone la introducción de novedades, tanto en la redacción como en el contenido:

(CA 6) De aquel que conpra o recibe en pennos alguna heredat, aquella misma fidança et los testigos, segunt del fuero, deuen seer de aquella uilla o es la heredat, et deuen saber et ueder aquella misma heredat, que

27.- María Antonia Martín Zorraquino y María Luisa Arnal Purroy, "Introducción al estudio lingüístico del Fuero de Jaca”, en El Fuero de Jaca, vol. II, Zaragoza, El Justicia de Aragón, 2003, pp. 319-351. 
por cierto sepan de qual heredat son fidanças et testigos, que si assi feito non fuere, el conprador o el que el pennal recibe puede a ella de cierto perderla, qual la fidança et los testigos non saben ont son fidanças o testigos. Exament, qui conpra o en pennos recibe, si non recibe testigos et fidança del heredat puede a ella por l'qual fallimiento perder, et si assi pudieren seer auidos los testigos o las fidanças del lugar o de la villa o fuere la heredat, seya auida. O si auer no la pudieren, seya auida del regno, de qual que lugar, seyan buenos et leales o de cruz.

Finalmente, sobre el texto romance de $C A$, se lleva a cabo una traducción literal, que será la versión latina oficial $(\mathrm{CH})$ :

(CH 2.11.4) De illo qui emit aut accepit in pignus haereditatem aliquam, ipsae fidantiae et testes, secundum forum, debent esse de ipsa villa ubi est haereditas, et debent scire et videre ipsam haereditatem, ut pro certo sciant de qua haereditate sunt fidantiae et testes, quod, si ita factum non fuerit, emptor, aut qui pignale accepit, potest de facili eam amittere, quia fidantiae aut testes nesciunt unde sunt fidantiae aut testes. Similiter, qui emit aut in pignus accipit, si non accipit testes et fidantiam de haereditatae, quam accipit, potest de facili eam amittere. Et si commode poterunt haberi testes vel fidancie de loco vel villa, ubi fuerit hereditas, habeantur; vel si haberi non poterunt, habeantur de regno, cuiuscumque loci sint, boni et legales, vel de cruce.

\section{b) La recopilación $R L$}

Con respecto a los capítulos contenidos en la recopilación $R L$, con frecuencia presentan un contenido muy similar al de $A$, con la sola diferencia del idioma en que están escritos. Es cierto que en ocasiones los capítulos de $R L$ están menos desarrollados que los de $A$ en relación con la Compilación de Huesca, pero también podemos encontrar algún capítulo en que $R L$ se muestra más cercano a la redacción de $C H$ que la versión romance de $A$ :

(A 9) Aquel qui es fiança per altre per auer o per altre cosa no pot esser razonador en aquel feyt metex per que es fiança.

(RL 8) Si quis fuerit fidanza pro aliquo homine pro peccunia aut pro alia qualibet causa, postea in eodem facto pro quo est fidanza, non potest esse aduocatus eius.

(CH 1.10.07) Si aliquis homo fuerit fidancia pro aliquo homine pro pecunia vel pro alia qualibet causa, postea in eodem facto pro quo est fidantia, non potest esse advocatus eius.

Por otro lado, aparentemente casi todos los capítulos de las recopilaciones $U$ y $R L$ tienen su equivalente en $A$, e incluso siguen el mismo orden. Podría decirse que se complementan, llenando dos grandes grupos del articulado de $A$, y rara vez se producen coincidencias de contenido entre ellas. Sin embargo, 
en algunas ocasiones sí encontramos dichas coincidencias, y son éstas las que aportan la información más interesante.

El contenido de los capítulos de $U$ 2, 5, 6, 52, 55, 58, 107, 123, 126, 64/109/154 equivale, respectivamente, al de los capítulos de $R L 10,34,26$, 9, 20, 41/42, 38, 55, 45, 33. Y en tales casos, mayoritariamente, el contenido de $A$ se halla más próximo al texto de $R L$, figurando luego en la Compilación de Huesca con algunos añadidos:

(RL 10) [...] De femina de uillis istud est fuerum: quod debet per suas dotes habere unam casam coopertam de duodecim biegas, et unam arenzatam de uinea, et unum campum de semenatura de uno arrobo de trico in uoce de linar, et suas joyas et uestes integre, et unum bonum lectum cum suis aparamentis, et duas bestias de arata cum suis complimentis, de melioribus scilicet bestiis que habebunt maritus et ipsa. Et in qualicumque hora habuerit prolem tantummodo et lucem uideat iam dicta mulier perdit suas dotes. In aliis causis que habuerunt accipiat suam partem sicut est fuerum.

(A 13) De femna uilana es atal fuer: que deu per ses dotz auer una casa cuberta de XII bigas e una arinçada de uinnas, e un campo de semnadura d'un arrouo de trigo en uoç de linar, e sos uestiduras entegrament $e$ sos ioyas, et un leyt ben parado dels millos draps que son en casa, e dos bestias d'arada de las millos de casa ab sos apareyllament. Pero si a criatura del marit, uiua la criatura o no, solament que ueya lum, la uilana pert sos dotz. En las altras cosas que an, prenga ella sa part asi com es fuer, e un iugo de bestias e un lit entegre.

(CH 5.03.04) Villana debet habere per suas dotes, unam domun coopertam, in qua sint XII bige, et unam arençatam vineam, et et unum campum, in quo possit seminare unam arovam tritici in voce linaris, et suas vestes integre, et suas ioyas, et unum lectum bene paratum de melioribus pannis, qui sint in domo, et duas meliores bestias de domo, aptas ad laborandum, cum omnibus suis aparamentis. Tamen, si susceperit a viro filium, solummodo quod vivus nascatur, amittit villana suas dotes, licet incontinenti moriatur. Et in aliis rebus, que in domo fuerint, habeat secundum forum similiter partem suam. Quod, si non habet domum de XII bigis, det aliam in qua plures bige sint ultra XII, et ipsa accipiat partem integre, et medietatem omnium mobilium indifferebter, et medietatem immobilium omnium, que ex quo ambo fuerunt, pariter sunt lucrati.

Es cierto que encontramos algún caso en que $A$ se muestra más cercano al texto de $U$, pero incluso entonces la Compilación de 1247 prefiere seguir la lectura de $R L$. Así ocurre en el siguiente capítulo, en que podemos observar que $C A$ se aleja de $A$ y vierte al romance con bastante fidelidad el texto de $R L$ :

(A 270) De testimonis que jxiren per sarrayn o per iudeu per qual que cosa que sarrayn o iudeu aya a probar et ayan a donar testimoni christian, puys que'l testimoni christian aura feyt ab iura, lo qui's clamaua del sarrayn o del iudeu no pot tornar aquell per nulla manera a bataylla de ferre o d'altra 
cosa, quar no es fuero, quar si christian testimoni un atal feyt auia torna, no s'en trobaria nengun christian que fes testimoni per sarrayn o per iudeu.

(RL 55) Inter christianum et sarracenum et iudeum non habet tornam de batalla locum, set unusquisque defendit se ab altero per iuram planam de omni facto, tam de percussionibus quam de aliis causis uniuersis. Verumtamen si christianus agit de aliquo facto contra iudeum et uoluerit probare quod obicit contra illum, necessarii sunt ei duo testes, christianus scilicet et iudeus. Judeus probat contra christianum de omni facto cum christiano et iudeo. Christianus probat contra sarracenum cum christiano et sarraceno. Similiter sarracenus probat de omni facto contra christianum cum sarraceno et christiano.

(CA 2) Torna a batalla no a lugar entre cristiano et jodio et moro, mas qual se defendiere del otro por propio sagrament en toda razon, enpero quiscuno jure por su ley. Enpero si el cristiano contra el jodio a carta sobre alguna cosa et lo quisiere prouar, por II testigos, et assaber cristiano et jodio son i menester. Et el jodio exament contra el cristiano, prueua con jodio et cristiano. Exament contra'l moro, en toda cosa prueua con cristiano et moro, et el contrario. El moro exa misma ment, prueua en toda cosa contra'l judio con moro et jodio.

Como ya se ha comprobado anteriormente, la redacción romance de $C A$ se traduce al latín, en esta ocasión de manera literal, y el proceso hace que $C H$ ya no presente el mismo texto latino que $R L$ :

(CH 2.10.07) Torna aut batalla non habet locum inter christianum et iudaeum, aut sarracenum, sed quisque defendit se de alio proprio sacramento in omni causa; tamen quod quilibet iuret secundum suam legem. Veruntamen, si christianus cum iudaeo habeat causam super aliqua re, et volverit illud probare, duo testes, scilicet, christianus et iudaeus sunt ibi necessarii. Iudeus similiter contra christianum, cum christiano et iudeo probat. Christianus similiter contra sarracenum in omni causa probat cum christiano et sarraceno, et e converso. Sarracenus similiter probat in omni causa contra iudaeum cum sarraceno et iudaeo, et e converso.

\section{c) Otras fuentes}

Existe también una serie de capítulos de la Compilación de Huesca que no tienen su equivalente en $U, R L$ ni $A$. Sin embargo, podemos encontrarlos en las redacciones más tardías, $C, D$ y $E$. Así sucede en el siguiente caso:

(CA 8) De aquestos cristianos que testimonian por jodios et por moros sobre qual quiere cosa, despues que aquel mismo cristiano jurare, despues por ninguna manera no lo podra el otro tornar a ninguna batalla.

(E 262) De testimonis que yssen per sarrazin o per iudeu per qual que cosa sia, ningun non lo pot tornar a bataylla ni es for, quar si les christians per tal testimoni auien a estar tornatz a batailla, iudeu nin sarrazin non trobaria testimoni christian. 
Además, hay un conjunto de capítulos que no aparece en ninguna de las redacciones que hemos considerado como posibles fuentes de la Compilación de Huesca. Tal es el caso de $C A 1, C A 3$ y $C A 10$. Alguno de ellos, sin embargo, se encuentra en el códice $A^{2}$, pero se trata de una adición o interpolación posterior de las que ya hemos hablado:

(CA 3) Muitas uegadas alguno de qual condicion que seya non quisiere fer testimonio a otro omne de qual condicion quiere que seya, el sennor o la cort de aquel lugar o habitare aquel testigo constrigan a el fer el testimonio.

(AD 20) Qvantas que uegadas algun, de qual quere condition [...] lo seynnor o la iusticia del logar on estara aquel testimoni lo forçara de fer testimoni de uerdat.

\section{Conclusiones}

A partir del cotejo de los diversos testimonios conservados, cabe extraer algunas conclusiones sobre las posibles fuentes utilizadas en la redacción de los fueros de 1247.

\section{a) Redacciones $A$ y $U$}

En primer lugar, parece probable que la redacción $A$ sea, de los testimonios conservados, el más estrechamente emparentado con la Compilación de Huesca, pues casi todos los capítulos de aquélla tienen su equivalente en ésta, y el contenido de sus párrafos se aproxima, más que en ninguna otra redacción, al texto de 1247.

También podemos advertir que la recopilación $U$ guarda estrecha relación con $A$ y, por tanto, con la Compilatio Minor. Sus 155 capítulos originales coinciden aproximadamente con amplios pasajes de la redacción $A$, e incluso conservan su mismo orden.

Lo más habitual es que el contenido de $U$ aparezca ampliado en $A$ y que el texto de la Compilación de Huesca introduzca, a su vez, algunas innovaciones. Además, todos los capítulos de $U$ que tienen su equivalente en el texto de 1247 se hallan también en $A$. Da la impresión de que $A$ se sitúa a medio camino entre el texto de $U$ y el de los Fueros de 1247. Ramos Loscertales ya había señalado, después de cotejar la recopilación $U$ con la versión $A^{l}$, que "la forma de redacción conservada por la recopilación es más antigua que la del texto lemosín $\left[A^{l}\right]$ ", añadiendo que "el contenido de la mayoría de los capítulos es el mismo [en ambos], siendo las variantes, por lo general, 
aclaratorias del sentido"28. Por su parte, Molho comprobó sobre casos concretos que $U$ suele presentar un texto menos desarrollado y preciso que el de $A$, admitiendo que este último sería una ampliación o refundición de fecha posterior $^{29}$. La mayor antigüedad de $U$ quedaría confirmada por el hecho de que presenta un texto menos desarrollado, e incluso por el uso de un latín bastante incorrecto mezclado con expresiones romances; pero también por la estrecha analogía que presenta con respecto a la llamada redacción $A^{p}$, en la que ya dijimos había visto Molho el vestigio de una redacción arcaica. Observemos el siguiente caso:

(A $\mathrm{A}^{\mathrm{p}}$ ) De espada uel de cuytiello qui lo trayt in villa muro çinta et ferit jnde, abet calonja quingentos sol., et si matat, mill sol. De alia ferida de pueno o de petra, $L X$ sol., cun testes que abeat. E si non ferit, no abet colonia vel torna auta que perdat illo pugno.

(U 103) De spata uel cultillo qui trahit in uilla muro cinta et ferit inde, habetur calonia D solidos; et si occidit, mille solidos. De alia ferida de pugno uel de petra, LX solidos, cum testibus quos habeat. Set si non ferit, non habet caloniam nec tornam uel quod perdat pugnum.

\section{b) La recopilación $R L$}

Con respecto a $R L$, ya dijimos que sus capítulos casi siempre presentan un contenido semejante al de $A$, con la sola diferencia del idioma en que están redactados. Sin embargo, mientras que en algunas ocasiones la redacción $A$ se encuentra más cerca de la Compilación de Huesca, en otros casos es $R L$ la que se muestra más próxima. Además, hay algunos capítulos de $R L$ que, teniendo su equivalente en $C H$, no se hallan en la redacción $A$. Se trata concretamente de los capítulos 11, 15, 36. Resulta, por tanto, plausible que ambas redacciones representen dos tradiciones independientes, aunque cronológicamente muy cercanas.

También hemos podido comprobar, en nuestro cotejo de los textos, que en aquellos casos en que $U$ y $R L$ contienen capítulos equivalentes, la redacción $A$ casi siempre presenta un texto más desarrollado, al igual que $R L$, lo cual parece confirmar la mayor cercanía cronológica de $A$ con respecto a $R L$, y de ambas con respecto al texto de los Fueros de Aragón. Sin embargo, en alguna ocasión $A$ se muestra más próxima a $U$, e incluso entonces la Compilación de Huesca sigue manteniéndose más cercana a $R L$, lo cual confirmaría que $A$ se halla en la misma tradición textual que $U$, frente a $R L$.

28.- J. M. Ramos Loscertales, “Textos para el estudio del derecho aragonés en la Edad Media”, Anuario de Historia del Derecho Español, II (1925), pp. 491-492.

29.- M. Molho, "Difusión”, pp. 347-349. 
Por supuesto, debemos ser sumamente cautelosos en este punto, por cuanto ya hemos visto que los textos pueden haber sido objeto de alteraciones posteriores a 1247. Pero, a la vista de los testimonios, sí podemos identificar dos fuentes utilizadas en la redacción de la Compilación de Huesca. Una de ellas estaría representada por $A$ y $U$, que se hallan estrechamente emparentadas, aunque reflejan seguramente dos momentos distintos en su evolución. La otra fuente quedaría representada por $R L$, que es un testimonio fragmentario de una redacción perdida. Porque seguramente $R L$, tal como defendió Molho ${ }^{30}$, sería parte de un texto mayor, quizá una amplia recopilación del derecho tradicional jaqués de la que se extraería $R L$ para adaptarla a los usos del valle del Ebro. Parece, por tanto, poco probable que $U$ y $R L$ fueran refundidas en A para formar una "recopilación de recopilaciones", tal como apuntó Ramos Loscertales $^{31}$, y mucho menos que formaran parte de las cuatro recopilaciones que imaginó el profesor Meijers ${ }^{32}$.

\section{c) Otras fuentes}

Ya hemos visto que en $C A$ se recogen también algunos capítulos que no aparecen en $A$ ni en ninguna de las recopilaciones $U$ y $R L$. Sí los encontramos, en cambio, en $C, D$ y $E$. Este hecho podría permitirnos señalar la existencia de, al menos, otra fuente distinta de las ya mencionadas, la cual se habría utilizado igualmente en la confección del texto de la Compilación de Huesca. Sin embargo, debemos considerar la posibilidad, ya apuntada más arriba, de que se trate de una interpolación posterior. No olvidemos que las redacciones $B, C, D$ y $E$ presentan un cierto ordenamiento sistematizado, a imitación de los Fueros de Aragón, lo cual las hace sospechosas.

Por otro lado, la existencia de capítulos en la Compilación de Huesca que no tienen correspondencia con ninguna de las redacciones emparentados con el derecho altoaragonés anterior a 1247 puede ser interpretada de diversas maneras. Podríamos suponer que se utilizaron fuentes de las que no ha quedado testimonio documental; puede tratarse también de capítulos creados ex novo en 1247; incluso podrían haberse tomado contenidos de una tradición legislativa transmitida de forma oral, hecho sobre el cual ya incidió Antonio Ubieto $^{33}$.

\footnotetext{
30.- Ibídem, p. 350.

31.- J. M. Ramos Loscertales, Fuero de Jaca, p. XVIII.

32.- E. M. Meijers, "Los fueros de Huesca y Sobrarbe", Anuario de Historia del Derecho Español, XVIII (1947), p. 37.

33.- Antonio Ubieto Arteta, "Los precedentes de los Fueros de Aragón”, en Vidal Mayor, Huesca, Excelentísima Diputación e Instituto de Estudios Altoaragoneses, 1989, pp. 23-41.
} 
Finalmente, todos estos materiales tan diversos quedaron aglutinados, durante las Cortes de 1247, en un solo texto romance $(C A)$, el cual fue aprobado e inmediatamente vertido al latín, convirtiéndose en la versión oficial de los Fueros de Aragón $(\mathrm{CH})$. Otra cosa es que, a lo largo de los siglos XIV y XV, fueran surgiendo diversas versiones romanceadas de la Compilatio Minor, pero éstas ya serían traducciones más o menos libres, muy divergentes entre sí, que estaban destinadas a facilitar la interpretación del texto, tal como expuse en su momento ${ }^{34}$. lación de Huesca, Zaragoza, El Justicia de Aragón, 1999. 